\title{
INVESTIGAÇÃO-AÇÃO SOBRE O VIH/SIDA EM ESTUDANTES DE ENFERMAGEM
}

\author{
Cláudia Chaves \\ Doutoranda em Ciências de Enfermagem \\ Instituto de Ciências Biomédicas Abel Salazar \\ SIGMA - Phi Xi Chapter / CI\&DETS / \\ Politécnico de Viseu - Portugal \\ claudiachaves21@gmail.com \\ João Duarte \\ UICISA:E / CI\&DETS \\ Politécnico de Viseu - Portugal \\ Paula Nelas \\ UICISA:E / CI\&DETS \\ Politécnico de Viseu - Portugal \\ Emília Coutinho \\ SIGMA - Phi Xi Chapter/ UICISA:E / CI\&DETS / \\ Politécnico de Viseu - Portugal \\ Carla Cruz \\ UICISA:E / CI\&DETS \\ Politécnico de Viseu - Portugal
}

Fecha de Recepción: 19 Mayo 2019

Fecha de Admisión: 25 Septiembre 2019

\section{RESUMO}

Enquadramento: Os conhecimentos, crenças e estereótipos acerca do VIH são um desafio para os estudantes de enfermagem, constituindo-se estes como um importante grupo para uma intervenção formativa. Objectivo do estudo: Verificar se a intervenção formativa acerca do VIH/SIDA influencia o nível de conhecimentos e estereótipos. Metodologia: Investigação-ação, com uma amostra de 30 estudantes do primeiro ano, intervenção formativa sobre o VIH/SIDA com follow-up após 3 meses. Para colheita de dados foi utilizado um questionário de caracterização sociodemográfica e a escala de avaliação de estereótipos sobre VIH/SIDA (SAAQ - "The Stereotypes About AIDS Questionnaire"). Resultados: No global verifica-se que os estudantes detêm conhecimentos sobre 0 vírus. No estudo verifica-se que após a intervenção formativa houve melhorias do conhecimento em nove das 15 dimensões analisadas, sendo que apenas a dimensão "sexualidade" é estatisticamente significativa $(p=0,015)$. Conclusões: 0 s estudantes de enfermagem apresentam níveis de conhecimento razoáveis, no entanto, face aos resultados, sugerimos mais formação ao longo da licenciatura.

Palavras-chave: estereótipos; conhecimentos; estudantes de enfermagem; VIH/SIDA. 


\section{ABSTRACT}

HIV/AIDS research-action in nursing students.

Background: Knowledge, beliefs and stereotypes about HIV are a challenge for nursing students, and these constitute an important group for a training intervention. Objective of the study: To verify if the training intervention on HIV / AIDS influences the level of knowledge and stereotypes. Methodology: Action research, with a sample of 30 first-year students, training intervention on HIV / AIDS with follow-up after 3 months. A sociodemographic characterization questionnaire and the Stereotypes About AIDS Questionnaire (SAAQ) scale were used to collect data. Results: In the global it turns out that the students have knowledge about the virus. The study shows that after the training intervention there were improvements in knowledge in nine of the 15 dimensions analyzed, with only the dimension "sexuality" being statistically significant $(p=0.015)$. Conclusions: Nursing students present reasonable levels of knowledge; however, in view of the results, we suggest more training during the undergraduate course.

Keywords: stereotypes; knowledge; nursing students; HIV / AIDS.

\section{INTRODUÇÃO}

Em Portugal as taxas de incidência de novos casos de infeção pelo vírus da imunodeficiência humana (VIH) são das mais elevadas da União Europeia (Portugal, 2018). Perante este facto é necessário que enfermeiros e estudantes de enfermagem estejam aptos para cuidar de pessoas que vivem com esta infeção ou venham a desenvolver a Síndroma de Imunodeficiência Adquirida (SIDA). É fundamental que haja educação formal de enfermeiros e estudantes de enfermagem sobre 0 VIH/SIDA, e especialmente, em estudantes de enfermagem antes do início da carreira profissional (Pickles, King, \& Belan, 2012). Os enfermeiros encontram-se na linha da frente para a prevenção do VIH/SIDA e são elementos chave no cuidar destes clientes (Bernhed, Mollstedt, \& Rosengren, 2018). Estudos anteriores mostraram que a educação relativa ao VIH/SIDA reduziu as atitudes negativas em relação às pessoas que vivem com VIH/SIDA (Genberg, Hlavka, Konda, et al, 2009; Suominen, Laakkonen, Polukova, et al, 2015; Khan, Bilal, \& Siddiqui, 2017; Feyissa, Lockwood, Woldie, Munn, 2019). A capacitação através de um conhecimento sólido e atitudes saudáveis faz com que as competências aprendidas ultrapassem o mero desenvolvimento profissional e sejam úteis para tomadas de decisão individual ao nível da vida pessoal e social. Por isto, é importante 0 estudo do conhecimento do VIH e SIDA entre os estudantes universitários. (Korhonen et al, 2012; Pickles, King, Belan, 2012).

Realçar ainda, que a intervenção dos enfermeiros não se centra apenas na sua vertente de prestação de cuidados, mas também, no seu papel preventivo, de apoio à sociedade, no seu esclarecimento e na sua formação (Williams, Wang, Burgess, et al, 2004; Stavropoulou, Stroubouki, Lionaki et al, 2011). Os planos de estudos dos currículos de formação em enfermagem devem reforçar a compreensão do significado de ser estigmatizado pela sociedade, e desenvolver estratégias facilitadoras para os estudantes de enfermagem em conciliar as suas crenças pessoais com os valores profissionais da enfermagem (Pickles, King, Belan, 2012; Pickles, Lacey, \& King, 2019).

Com 0 aumento generalizado da população infetada com o VIH/SIDA, é preocupante o défice de conhecimentos e os mitos presentes na população, nomeadamente nos futuros profissionais de saúde, que possivelmente serão os cuidadores das pessoas infetadas. É neste contexto que emerge a questão de investigação: Qual o nível de conhecimentos e estereótipos acerca da infeção pelo vírus VIH/SIDA em estudantes de enfermagem? E a partir desta colocaram-se as seguintes questões de investigação secundárias: De que modo as variáveis sociodemográficas influenciam os conhecimentos e estereótipos sobre o VIH/SIDA dos estudantes de enfermagem? Qual a influência da formação nos conhecimentos e estereótipos sobre o VIH/SIDA? Para a consecução destas ques- 
tões delinearam-se os seguintes objectivos: caracterizar a influência das variáveis sociodemográficas nos conhecimentos e estereótipos sobre o VIH/SIDA; verificar se a intervenção formativa acerca do VIH/SIDA influencia o nível de conhecimentos e estereótipos sobre o VIH/SIDA.

Para tal optou-se pela realização de uma investigação-ação que avalia os conhecimentos, estereótipos e crenças dos estudantes de enfermagem do primeiro ano antes e após uma formação sobre VIH/SIDA lecionada a esses mesmos estudantes.

\section{MATERIAL E MÉTODOS}

0 estudo desenvolvido tipifica-se nos estudos quantitativos. Investigação-ação, com uma amostra não probabilística por conveniência de 30 estudantes do primeiro ano do curso de enfermagem, intervenção formativa sobre o VIH/SIDA com follow-up após 3 meses.

Para este estudo foi utilizado como instrumento de colheita de dados um questionário de caracterização sociodemográfica e a escala de SAAQ - "The Stereotypes About AIDS Questionnaire" (Snell, William, Finney, Godwin, 1991). 0 protocolo de avaliação construído para o efeito é constituído por doze questões de carácter sociodemográfico e a escala SAAQ apresenta quatro categorias de estereótipos relacionados ao VIH/SIDA. Dentro do primeiro grupo são avaliados 30 itens; no segundo grupo são avaliados 35 itens; no terceiro são avaliados 30 e, no quarto e último grupo, são 20 itens. Para a seleção dos diversos estereótipos sobre o VIH/SDA são mensuradas quatro categorias de estereótipos relacionados ao VIH/SIDA: (A) Crenças estereotipadas globais sobre 0 VIH/SIDA, (B) As atitudes pessoais sobre o VIH/SIDA, (C) Questões médicas sobre o VIH/SIDA e (D) Questões sexuais sobre 0 VIH/SIDA. Cada uma destas escalas apresenta várias subescalas. Os itens na secção A do SAAQ (Estereótipos globais sobre o VIH/SIDA) apresentam quatro subescalas separadas focadas nos estereótipos sobre: (1) A necessidade de educação relacionada com o VIH/SIDA; (2) A confidencialidade e 0 VIH/SIDA; (3) A transmissão do VIH/SIDA e (4) 0 VIH/SIDA é causado pela homossexualidade.

Os itens na secção B do SAAQ (Atitudes pessoais sobre o VIH/SIDA) apresentam cinco subescalas separadas focadas nos estereótipos sobre: (1) 0 desejo de evitar os que estão afetados com 0 VIH/SIDA; (2) 0 VIH/SIDA não é compreendido como relevante para o próprio; (3) A mentalidade estreita sobre o VIH/SIDA; (4) A questão do VIH/SIDA está a ser exagerada e (5) A ideia de que 0 VIH/SIDA é uma punição moral.

Os itens na secção C do SAAQ (Questões médicas sobre o VIH/SIDA) apresentam quatro subescalas separadas focadas nos estereótipos sobre: (1) A crença de que a SIDA é uma ameaça para os profissionais da saúde; (2) Proteger o sistema de abastecimento de sangue de Portugal do vírus do VIH/SIDA; (3) Uma cura para o VIH/SIDA e (4) 0 teste do VIH/SIDA deveria ser realizado. Os itens na Secção D do SAAQ (Questão Sexual sobre o VIH/SIDA) apresentam duas subescalas separadas focadas nos estereótipos sobre: (1) A relação entre o VIH/SIDA e atividade sexual e (2) A prevenção do VIH/SIDA através do uso de preservativos.

Em cada um dos itens, os indivíduos devem indicar o quanto eles concordam ou discordam do enunciado. Para isto, foi utilizado um formato de 5 pontos de Likert: concordo (2); concordo ligeiramente (1); não concordo nem discordo (0); discordo ligeiramente (-1); e discordo (-2). A fim de criar scores nas subescalas, os itens em cada subescala são médias. Os scores mais altos positivos (negativo) correspondem à maior concordância (desacordo) com os estereótipos medidos pelo SAAQ (Snell, Finney \& Godwin, 1998). Este questionário tem uma estimativa de 35 a 45 minutos para ser concluído. Vários itens da Stereotypes About AIDS Scale (SAAS) são revertidos-marcados (A8, A20, A21, C2, C12, e D4) antes das subescalas são computados.

Os autores da escala descobriram que para a categoria A - Crenças estereotipadas sobre 0 
VIH/SIDA do SAAQ, a fiabilidade varia de um mínimo de 0,75 para um máximo de 0,85; para a categoria $B$ - As atitudes pessoais sobre o VIH/SIDA, a fiabilidade varia de um mínimo de 0,72 para um máximo de 0,87; para a categoria $\mathrm{C}$ - Questões médicas sobre $0 \mathrm{VIH} / \mathrm{SIDA}$, a fiabilidade varia de um mínimo de 0,64 para um máximo de 0,83 e para a categoria D - Questões sexuais sobre o VIH/SIDA, 0 alfa de Cronbach foi respetivamente de 0,86 a 0,78 .

Este questionário foi devidamente adaptado e traduzido por peritos do idioma inglês. Os procedimentos de tradução e retroversão permitiram constatar que não existem diferenças impeditivas da utilização deste instrumento, exceto na secção VIH/SIDA II no item 13 - "Apenas as pessoas da Califórnia foram afetadas pelo VIH/SIDA", no item 21 - "O VIH/SIDA pode, eventualmente, levar 0 Sistema de Saúde dos Estados Unidos à falência" e item 30 - "Quem habita em São Francisco, tem maior probabilidade de contrair o VIH/SIDA do que qualquer outra pessoa".

Após ser concluída a tradução e adaptação dos questionários, e obtida a aprovação da Comissão de Ética da Escola Superior de Saúde de Viseu (Parecer № 03/2016), iniciou-se a aplicação dos questionários em sala de aula, contando com a colaboração dos docentes. Dentro dos procedimentos éticos, os questionários foram aplicados esclarecendo previamente 0 seu objetivo e funcionamento, não recorrendo a práticas de coação, permitindo assim a livre manifestação de vontade pessoal dos estudantes para aderirem ao estudo. Do mesmo modo, os estudantes foram informados de que os questionários eram anónimos. Para que os participantes entendessem o objetivo do instrumento de avaliação e a importância do rigor das respostas, foi acrescentado na primeira página do instrumento um breve texto de apresentação com essas explicações, assim como 0 agradecimento pela colaboração. Antes da aplicação deste instrumento de avaliação, realizou-se a descrição dos objetivos, procedimentos e instrumentos a serem utilizados.

Seguindo a metodologia da investigação ação, foi realizada uma intervenção formativa no âmbito do VIH/SIDA, após terem sido aplicados os questionários. A formação foi realizada através do método expositivo. Após a realização da formação, decidiu-se com a equipa de investigação que 0 tempo de follow-up seria de três meses, de forma a comparar os conhecimentos sobre VIH/SIDA antes e após a formação. Efetuada a recolha de dados, estes foram lançados e processados no programa de estatística SPSS (Statistical Package for the Social Sciences) versão 23.0 para o Windows. Para o tratamento estatístico recorreu-se à estatística descritiva e inferencial. Determinou-se as frequências absolutas e percentuais, médias, amplitude de variação, coeficiente de variação e desvio padrão, como também medidas de assimetria e achatamento. Na estatística inferencial, utilizou-se o Teste t de Student, Teste Binomial e o Teste de Mann-Whitney.

\section{RESULTADOS}

Relativamente ao sexo da nossa amostra, esta é apenas constituída por estudantes do sexo feminino, correspondendo assim a 100,0\% da amostra. As estudantes apresentam uma idade mínima de 18 e uma máxima de 26 anos, a que corresponde a uma idade média de 18,8 anos $( \pm 1,5$ anos). Idades inferiores a 18 anos representam $53,3 \%$ da totalidade da amostra e idades superiores a 19 anos representam 46,7\%. Verificou-se que a maioria das estudantes vive na cidade $(70,0 \%)$. A maioria das estudantes reside com outros/amigos/colegas/residência estudantil $(63,3 \%)$ e $37,6 \%$ habitam com os pais/família. Verificou-se que $60,0 \%$ dos pais possuem como habilitações literárias o 9o ano de escolaridade, $26,7 \%$ ensino superior e 13,3\% frequentou 0 ensino secundário. No que diz respeito às habilitações literárias da mãe verificou-se que a maior percentagem frequentou 0 ensino superior (43,3\%), até ao $9^{\circ}$ ano de escolaridade registaram-se $33,3 \%$ e com 0 ensino secundário, 23,3\% das mães.

Quando foram questionadas as estudantes se durante o seu percurso escolar tiveram alguma 
formação sobre VIH/SIDA, a maioria $(90,0 \%)$ não teve/frequentou nenhuma formação nesta área, enquanto que apenas 10,0\% afirmou ter tido formação no secundário.

Procurou-se estudar a relação entre a idade e os conhecimentos sobre VIH/SIDA tendo-se efetuado 0 teste de Mann-Whittney. Os resultados revelam que os estudantes com idade inferior ou igual a 18 anos apresentam melhores conhecimentos sobre "educação", "homossexualidade", "irrelevância para o próprio", "mentalidade", "cura", "testes" e "preservativo", enquanto que os melhores conhecimentos, nos indivíduos de idade superior ou igual a 19 anos apresentam-se nas respostas em relação à "confidencialidade", "evitar", "exagero", "moralidade", "questões médicas", "banco de sangue" e "sexualidade". No entanto, as diferenças entre os grupos etários estatisticamente mais significativas só estão presentes na variável independente relativa ao "exagero"com um valor de significância ( $p=0,002)$, como se pode observar na tabela 1 .

Tabela 1- Teste U de Mann-Whitney para diferença de médias dos conhecimentos sobre o VIH/SIDA em função da idade

\begin{tabular}{c|c|c|c|c}
\hline Idade & $<=\mathbf{1 8}$ anos & $>=\mathbf{1 9}$ anos & $\mathbf{U M W}=\mathbf{Z}$ & $\mathbf{p}$ \\
\hline Variáveis & $\mathbf{O M}$ & $\mathbf{O M}$ & & \\
\hline Educação & 16,50 & 14,36 & $-0,68$ & 0,497 \\
Confidencialidade & 15,19 & 15,86 & $-0,21$ & 0,835 \\
Transmissão & 15,25 & 15,79 & $-0,17$ & 0,867 \\
Homossexualidade & 15,63 & 15,36 & $-0,11$ & 0,915 \\
Evitar & 14,53 & 16,61 & $-0,65$ & 0,515 \\
Irrelevância para o & 16,19 & 14,71 & $-0,49$ & 0,627 \\
próprio & 15,53 & 15,46 & $-0,02$ & 0,982 \\
Mentalidade & 10,94 & 20,71 & $-3,07$ & 0,002 \\
fechada & 14,66 & 16,46 & $-0,76$ & 0,449 \\
Exagero & 13,69 & 17,57 & $-1,21$ & 0,225 \\
Moralidade & 14,63 & 16,50 & $-0,60$ & 0,548 \\
Questões Médicas & 15,97 & 14,96 & $-0,32$ & 0,752 \\
Banco de Sangue & 16,06 & 14,86 & $-0,38$ & 0,701 \\
Cura & 17,03 & 13,75 & $-1,21$ & 0,224 \\
Testes & 14,81 & 16,29 & $-0,46$ & 0,645 \\
Preservativo & & &
\end{tabular}

Relativamente à relação entre a variável "onde vive no tempo de aulas" e a variável independente que apresenta um índice mais elevado, temos no grupo que vive em ambiente rural a variável independente "a confidencialidade relacionada com o VIH/SIDA", com uma ordem média de 18,67 e no grupo que vive em ambiente urbano é a variável "a necessidade de educação relacionada com 0 VIH/SIDA" com uma ordem média de 17,26. As diferenças entre os grupos não são estatisticamente significativas. 


\section{INVESTIGAÇÃO-AÇÃO SOBRE 0 VIH/SIDA EM ESTUDANTES DE ENFERMAGEM}

Os resultados revelam que relativamente à relação entre a variável "com quem vive durante as aulas" e a variável independente que apresenta um índice mais elevado, temos no grupo "outras" a variável independente "a necessidade de educação relacionada com o VIH/SIDA", com uma ordem média de 18,79 e no grupo "pais/família" é a variável "o VIH/SIDA não é compreendido como relevante para o próprio" com uma ordem média de 17,50, sendo que existem diferenças estatisticamente significativas entre os grupos na variável "a necessidade de educação relacionada com 0 VIH/SIDA" ( $p=0,006)$ (Tabela 2).

Tabela 2- Teste U de Mann-Whitney para diferença de médias dos conhecimentos sobre o VIH/SIDA em função da coabitação durante as aulas

\begin{tabular}{c|c|c|c|c}
\hline Coabita & Outras & Pais/Família & UMW=Z & p \\
\hline Variáveis & OM & OM & & \\
\hline Educação & 18,79 & 9,82 & $-2,75$ & 0,006 \\
Confidencialidade & 15,11 & 16,18 & $-0,32$ & 0,746 \\
Transmissão & 13,92 & 18,23 & $-1,29$ & 0,194 \\
Homossexualidade & 15,26 & 15,91 & $-0,25$ & 0,803 \\
Evitar & 14,89 & 16,55 & $-0,50$ & 0,617 \\
Irrelevância para o & 14,34 & 17,50 & $-1,00$ & 0,314 \\
próprio & 16,37 & 14,00 & $-0,79$ & 0,429 \\
Mentalidade & 16,71 & 13,41 & $-1,00$ & 0,317 \\
fechada & 15,79 & 15,00 & $-0,32$ & 0,749 \\
Exagero & 17,50 & 12,05 & $-1,65$ & 0,100 \\
Moralidade & 16,24 & 14,23 & $-0,62$ & 0,533 \\
Questões Médicas & 15,34 & 15,77 & $-0,13$ & 0,896 \\
Banco de Sangue & 15,82 & 14,95 & $-0,27$ & 0,791 \\
Cura & 15,97 & 14,68 & $-0,46$ & 0,644 \\
Testes & 15,29 & 10,68 & $-2,29$ & 0,022 \\
Preservativo & & &
\end{tabular}

Relativamente às habilitações literárias do pai dos estudantes, constata-se que as estudantes com pais que têm até ao 9ªno de escolaridade apresentam menos conhecimentos em geral, porém no que diz respeito às variáveis: "mentalidade fechada", "cura" e "testes" detêm maiores ordenações médias, ou seja, respostas com melhores resultados. As estudantes filhas de progenitores do sexo masculino com escolaridade até ao secundário são as que têm maiores conhecimentos no global, nas variáveis "educação", "confidencialidade", "homossexualidade", "exagero", "questões médicas", "banco de sangue", "preservativo" e "sexualidade". No que diz respeito aos conhecimentos das estudantes, com pais com habilitações até ao ensino superior, verifica-se que as variáveis com ordenações médias mais elevadas são a "transmissão", "irrelevância para o próprio" e "moralidade". No entanto, só se verifica diferença significativa na variável "sexualidade" $(p=0,026)$, ou 
seja, apenas esta influencia os conhecimentos das estudantes em função da escolaridade dos progenitores do sexo masculino.

Os resultados referentes às habilitações literárias da mãe das estudantes, constata-se que as estudantes com mães que têm até ao 90ano de escolaridade apresentam menos conhecimentos em geral, porém no que diz respeito às variáveis: "transmissão", "cura" e "testes" detêm maiores ordenações médias. As estudantes filhas de progenitores do sexo feminino com escolaridade até ao secundário são os que têm maiores conhecimentos no global, nas variáveis "educação", "confidencialidade", "evitar", "irrelevância para o próprio", "mentalidade fechada", "exagero", "questões médicas", "banco de sangue", "preservativo" e "sexualidade". E no que diz respeito aos conhecimentos das estudantes, com mães com habilitações até ao ensino superior, verifica-se que as variáveis com ordenações médias mais elevadas são a "homossexualidade" e "moralidade". No entanto, só se verifica diferença esta estatisticamente significativa na variável "preservativo" ( $p=0,043)$, ou seja, apenas esta influencia os conhecimentos das estudantes em função da escolaridade das mães.

Na tabela 3, observa-se o teste t efetuado para amostras emparelhadas no intuito de determinar se a intervenção formativa influenciou os níveis de conhecimento sobre a SIDA observados no primeiro e segundo momentos de avaliação. Verificamos que houve melhorias do conhecimento em nove das variáveis analisadas: "transmissão", "irrelevância para o próprio", "exagero", "questões médicas", "banco de sangue", "cura", "testes", "preservativo" e " sexualidade". Os valores de r apresentam correlação negativa para as variáveis "educação" "confidencialidade", "homossexualidade", "evitar", "irrelevância para o próprio", "mentalidade fechada" e "exagero", o que significa que entre o primeiro e o segundo momento de avaliação os fenómenos ocorreram em sentido inverso. No entanto, verifica-se que apenas a variável "sexualidade" apresenta valor estatisticamente significativo $(p=0,01)$. 
Tabela 3 - Teste t emparelhado para as diferenças de médias entre as variáveis do questionário do momento antes e após a formação

\begin{tabular}{|c|c|c|c|c|c|}
\hline Variáveis & $\neq$ médias & dp & $\mathbf{t}$ & $\mathbf{p}$ & $\mathbf{r}$ \\
\hline $\begin{array}{l}\text { Educação1 vs } \\
\text { Educação2 }\end{array}$ & 0,33 & 0,36 & 0,49 & 0,624 & $-0,04$ \\
\hline $\begin{array}{l}\text { Confidencialidade1vs } \\
\text { Confidencialidade } 2\end{array}$ & 0,03 & 0,71 & 0,22 & 0,824 & $-0,13$ \\
\hline $\begin{array}{c}\text { Transmissão1 } \\
\text { vsTransmissão2 }\end{array}$ & $-0,06$ & 0,97 & $-0,34$ & 0,733 & 0,07 \\
\hline $\begin{array}{l}\text { Homossexualidade1vs } \\
\text { Homossexualidade } 2\end{array}$ & 0,02 & 0,58 & 0,24 & 0,805 & $-0,07$ \\
\hline Evitar1 vs evitar2 & 0,01 & 0,88 & 0,10 & 0,919 & $-0,35$ \\
\hline $\begin{array}{l}\text { Irrelevância para o } \\
\text { próprio1vs } \\
\text { Irrelevância para o } \\
\text { próprio2 }\end{array}$ & $-0,15$ & 1,20 & $-0,68$ & 0,501 & $-0,22$ \\
\hline $\begin{array}{l}\text { Mentalidade fechada1 } \\
\text { vs Mentalidade } \\
\text { fechada2 }\end{array}$ & 0,11 & 0,59 & 1,02 & 0,316 & $-0,06$ \\
\hline $\begin{array}{l}\text { Exagero1 vs } \\
\text { Exagero2 }\end{array}$ & $-0,01$ & 0,78 & $-0,11$ & 0,909 & $-0,00$ \\
\hline $\begin{array}{c}\text { Moralidade1vs } \\
\text { Moralidade2 }\end{array}$ & 0,08 & 0,38 & 1,27 & 0,211 & 0,31 \\
\hline $\begin{array}{l}\text { Questões médicas1 vs } \\
\text { Questões médicas2 }\end{array}$ & $-0,11$ & 0,86 & $-0,74$ & 0,465 & 0,09 \\
\hline $\begin{array}{l}\text { Banco de sanguel vs } \\
\text { Banco de sangue } 2\end{array}$ & $-0,00$ & 0,44 & $-0,06$ & 0,946 & 0,33 \\
\hline Cura1 vs Cura2 & $-0,46$ & 1,45 & $-1,75$ & 0,090 & $-0,04$ \\
\hline Testes 1 vs Testes 2 & $-0,33$ & 1,72 & $-1,05$ & 0,298 & $-0,25$ \\
\hline $\begin{array}{l}\text { Preservativo1 vs } \\
\text { Preservativo2 }\end{array}$ & $-0,07$ & 0,37 & $-1,07$ & 0,291 & $-0,27$ \\
\hline $\begin{array}{l}\text { Sexualidade1 vs } \\
\text { Sexualidade2 }\end{array}$ & $-0,24$ & 0,50 & $-2,60$ & 0,015 & $-0,11$ \\
\hline
\end{tabular}

\section{DISCUSSÃO}

Como discussão metodológica importa realçar que encontramos como limitações, relativamente ao número da nossa amostra de estudantes que foi reduzido e ao questionário que por ser extenso, tornou-se exaustivo.

Os nossos resultados mostram melhores conhecimentos nos estudantes com pais com habilitações académicas mais altas. Isto é consistente com investigações anteriores, como é referido por numa investigação com estudantes de escolas indianas, desenvolvida por Selvan, Ross, Kapadia, Mathai e Hira (2001), onde se verificou que existe uma relação entre a manifestação de comportamentos sexuais de risco e o nível das habilitações académicas dos pais. Ou seja, verificou-se que 
os jovens cujos pais possuem um maior nível de escolaridade são aqueles que incorrem em menores comportamentos de risco, nomeadamente, no que se refere ao início da atividade sexual em idade precoce. É interessante perceber, pelos resultados estatisticamente significativos do estudo, que as habilitações literárias das mães dos estudantes que influenciam nos maiores conhecimentos do VIH/SIDA são aqueles sobre a variável "preservativo", ou seja, sobre os métodos de prevenção e, por conseguinte, a forma para diminuir os comportamentos de risco. No que diz respeito à relação entre a escolaridade dos pais dos estudantes e os conhecimentos sobre 0 VIH/SIDA, os melhores resultados apresentados são na variável "sexualidade". Em ambos os casos, a comunicação parental é essencial para a diminuição dos comportamentos de risco e para a promoção da saúde sexual e prevenção do VIH/SIDA. Verificamos que houve melhorias do conhecimento após a formação, resultado corroborado em outros estudos (Frain, 2017; Ngcobo, \& Mchunu, 2019).

\section{CONCLUSÕES}

A intervenção formativa teve influência nos conhecimentos das estudantes, principalmente na variável da sexualidade, sendo esta a única que mostrou melhorias estatisticamente significativas após a formação. Neste estudo as estudantes de enfermagem apresentam níveis de conhecimento razoáveis sobre o VIH/SIDA. Assim, considera-se que necessário uma atualização periódica dos conhecimentos nos estudantes de enfermagem em relação à infecção VIH. Sendo fundamental para o exercício profissional em enfermagem, nas áreas de intervenção da promoção do conhecimento, no aconselhamento na saúde sexual e reprodutiva, na educação e informação para uma vida saudável. Em simultâneo os próprios estudantes beneficiam, tendo atitudes mais conscientes perante 0 risco da infecção e o estigma a ela associado. Ficam mais hábeis para superar a barreiras, reduzem 0 défice de conhecimento e consequente 0 estigma, ao longo da formação pré-graduada. A formação relativa à infecção VIH / SIDA deve ser programada, modular, contínua e com uma abordagem ao longo do ciclo vital, desde a gravidez ao envelhecimento. Assim, é viável alcançar e manter 0 conhecimento, e uma atitude positiva perante as pessoas infetadas com 0 vírus VIH/SIDA. Um programa curricular em enfermagem tem de estar adequado à realidade epidemiológica das comunidades, e a atualização destes conteúdos de acordo com as metas nacionais e internacionais no combate à infecção, para que os estudantes enriqueçam o seu conhecimento e ganhem competências nesta área.

\section{REFERÊNCIAS BIBLIOGRÁFICAS}

Bernhed, J., Mollstedt, M., \& Rosengren, K. (2018). Dare to Care: A Qualitative Content Analysis Regarding Nursing Students' View on HIV in Vietnam. Home Health Care Management \& Practice, 30(4), 187-194. https://doi.org/10.1177/1084822318787773

Feyissa, G. T., Lockwood, C., Woldie, M., Munn, Z. (2019). Reducing HIV-related stigma and discrimination in healthcare settings: A systematic review of quantitative evidence. PLOS ONE 14(1): e0211298. https://doi.org/10.1371/journal.pone.0211298

Frain, J. A. (2017). Preparing every nurse to become an HIV nurse. Nurse Educ Today, 48, 129-33. https://doi.org/10.1016/j.nedt.2016.10.005

Genberg, B. L., Hlavka, Z., Konda, K. A., Maman, S., Chariyalertsak, S., Chingono, A., ... Celentano, D. D. (2009). A comparison of HIV/AIDS-related stigma in four countries: negative attitudes and perceived acts of discrimination towards people living with HIV/AIDS. Social science \& medicine (1982), 68(12), 2279-2287. doi:10.1016/j.socscimed.2009.04.005

Khan, R., Bilal, A., \& Siddiqui, S. H. (2017). Knowledge about HIV and Discriminatory Attitudes toward People Living with HIV in Pakistan. DHS Working Paper No. 134. Rockville, Maryland, USA: ICF. https://dhsprogram.com/pubs/pdf/WP134/WP134.pdf 
Korhonen, T., Kylma, J., Houtsonen, J., Valimaki, M., \& Suominen, T. (2012). University Students' Knowledge of, and Attitudes Towards, HIV and Aids, Homosexuality and Sexual Risk Behaviour: A Questionnaire Survey in Two Finnish Universities. (4. p. Journal of Biosocial Science, Compilador) doi:10.1017/S0021932012000338

Williams, A.B., Wang, H.W., Burgess, J., Wu, C., Gong, Y., Li, Y. (2006). Effectiveness of an HIV/AIDS educational programme for Chinese nurses. J. Adv. Nurs., 53 (6), 710-720, https://doi.org/10.1111/j.1365-2648.2006.03777.x

Ngcobo, S.J. \& Mchunu, G.G. (2019). 'Bachelor of Nursing students' HIV and AIDS knowledge in KwaZulu-Natal province: An evaluation study', Curationis 42(1), a1928. https://doi.org/10.4102/curationis.v42i1.1928

Pickles, D., King, L., Belan, I. (2012). Undergraduate nursing students' attitudes towards caring for people with HIV/AIDS. Nurse Educ. Today, 32, 15-20. doi:10.1016/j.nedt.2011.01.008

Pickles, D., Lacey, S. de, \& King, L. (2019). Conflict between nursing student's personal beliefs and professional nursing values. Nursing Ethics, 26(4), 1087-1100. https://doi.org/10.1177/0969733017738132

Portugal. Ministério da Saúde. Direção-Geral da Saúde (2018). Infeção VIH e SIDA Desafios e Estratégias 2018. Lisboa: Direção-Geral da Saúde https://www.sns.gov.pt/wpcontent/uploads/2018/07/RelatorioVIH_SIDA2018.pdf

Selvan, M. S., Ross, M. W., Kapadia, A. S., Mathai, R., \& Hira, S. (2001). Study of perceived norms, beliefs and intended sexual behaviour among higher secondary school students in India. AIDS Care - Psychological and Socio-Medical Aspects of AIDS/HIV, 13(6), 779-788. https://doi.org/10.1080/09540120120076968

Snell, W.E., Finney P.D., \& Godwin L. J. (1998). The Stereotypes About AIDS Scale. In: Handbook of Sexuality-related Measures. 1st ed. SAGE Publications, Inc.

Snell, Jr., William, E.; Finney, P.; Godwin, L.J. (1991). AIDS Questionnaire: Stereotypes [SAAQ]. IN: UCLA Center for HIV Identification, Prevention and Treatment Services database. (Retrieved 8/2007). http://chipts.ucla.edu/resources/download-info/stereotypes-about-aids-questionnairesaaq/

Stavropoulou, A., Stroubouki, T., Lionaki, A., Lionaki, S., Bakogiorga, H., Zidianakis, Z. (2011). Student Nurses' perception on caring for people living with HIV. Health Sci J. 5, 288-96. https://pdfs.semanticscholar.org/2a1d/5b0ce64febb9449580e66688d95f0abb208f.pdf

Suominen, T., Laakkonen, L., Polukova, M., Nikolaenko, S., Lipiainen, L., \& Valimaki, M. (2015). Russian nursing students knowledge level ant attitudes in the context of human immunodeficiency virus (HIV) - a descreptive study. http://www.ncbi.nlm.nih.gov/pubmed/25598706 